\title{
Study of the Fluctuations of Net-charge and Net-protons Using Higher Order Moments
}

\author{
Tapan K. Nayak ${ }^{a}$ for the STAR collaboration \\ ${ }^{a}$ Variable Energy Cyclotron Centre, 1/AF Bidhan Nagar, Kolkata - 700064, India
}

\begin{abstract}
We present the STAR preliminary results on mid-rapidity and low transverse momentum mean, standard deviation, skewness, and kurtosis of net-charge and net-proton distributions in $\mathrm{Au}+\mathrm{Au}$ and $\mathrm{Cu}+\mathrm{Cu}$ collisions at $\sqrt{s_{N N}}=200 \mathrm{GeV}$ for various collision centralities. All the measured high moments of these distributions can be scaled by the number of participating nucleons, consistent with the soft process emissions. The ratios of fourth to second order cumulants of both the net-charge and net-proton distributions are consistent with models without QCD critical point.
\end{abstract}

\section{Introduction}

One of the goals of the physics program at the Relativistic Heavy-Ion Collider (RHIC) is to locate a critical point in the QCD phase diagram [1]. The QCD phase diagram is often plotted as temperature $(T) v s$. baryon chemical potential $\left(\mu_{\mathrm{B}}\right)$. Lattice Gauge Theory (LGT) calculations indicate that at small chemical potential $\left(\mu_{\mathrm{B}} \sim 0\right)$, corresponding to the cases for RHIC and LHC, the transition from hadronic to partonic matter is a cross-over [2]. On the other hand, model calculations suggest that when the $\mu_{\mathrm{B}}$ is larger, the transition becomes first order in nature [3]. Therefore, one expects the existence of a critical point at the end of first order transition [1]. At RHIC we plan to scan [4] the center of mass energy $\left(\sqrt{s_{N N}}\right)$ to vary $\mu_{\mathrm{B}}$ in order to search for the location of the QCD critical point. Several lattice calculations suggest the existence of the critical point for $\mu_{B}>160 \mathrm{MeV}$ [5, 6], corresponding to a center of mass energy of $\sqrt{s_{N N}}<20 \mathrm{GeV}$.

One of the characteristic signatures of the critical point is an increase in fluctuations of various event-by-event observables [7, 8]. The moments of the conserved quantities such as netcharge, net-baryon and net strangeness are related to respective thermodynamic quantity susceptibilities. The LGT calculations, indeed, have shown that the fourth moment of these distributions have large values or diverge at critical temperature near the QCD critical point [5, 9, 10, 11]. It has been proposed that higher moments of event-by-event net-charge and net-proton multiplicities are significantly more sensitive to existence of critical point compared to measures based on second moments [12]. The fourth moments of these multiplicity distributions are expected to be proportional to the seventh power of the correlation length [12]. It is expected that the evolution of fluctuations from the critical point to the freeze-out may lead to non-Gaussianity in the eventby-event multiplicity distributions. Thus, the kurtosis of multiplicity distributions (net-charge or net-proton) could be a sensitive observable in the search of QCD critical point.

We present the results of the moments of net-charge and net-proton distributions at midrapidity from $\mathrm{Au}+\mathrm{Au}$ and $\mathrm{Cu}+\mathrm{Cu}$ collisions at $\sqrt{s_{N N}}=200 \mathrm{GeV}$ in the STAR experiment at RHIC. 


\section{Analysis Details, Results and Discussions}

With the large acceptance and excellent particle identification capabilities, STAR provides the best environment for studying event by event fluctuations. The charged particles were detected in the Time Projection Chamber (TPC) and protons were identified by their specific energy loss in the TPC gas. Standard STAR track quality cuts were applied in this analysis. The charged particle tracks are chosen to be within the transverse momentum $\left(p_{T}\right)$ range of $0.15-1.0 \mathrm{GeV} / \mathrm{c}$ whereas for protons the $p_{T}$ range is taken to be $0.4-0.8 \mathrm{GeV} / \mathrm{c}$ in order to have a clean proton identification and to reject secondary protons from interactions with detector material. The analysis is carried out for $\mathrm{Au}+\mathrm{Au}$ and $\mathrm{Cu}+\mathrm{Cu}$ collisions at $\sqrt{s_{N N}}=200 \mathrm{GeV}$ for various collision centralities.
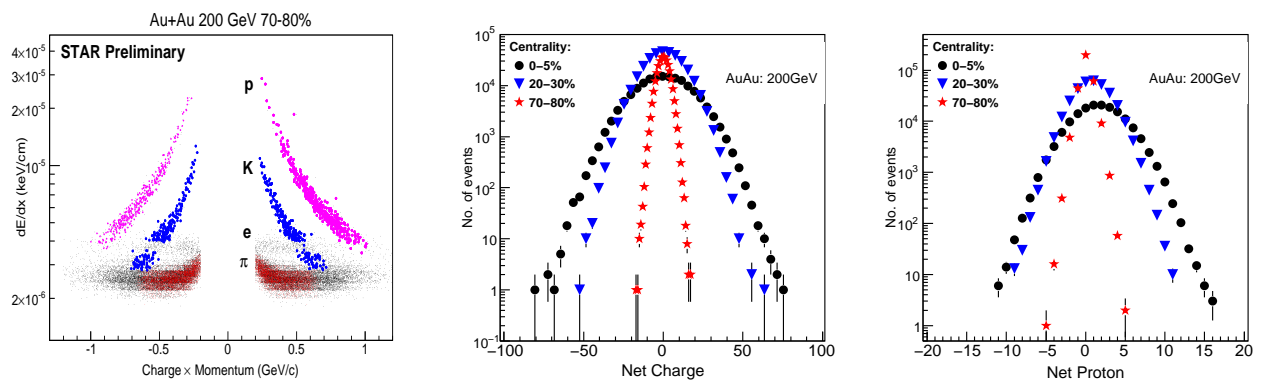

Figure 1: Left panel shows the $d E / d x$ (specific ionization loss) $v s$. rigidity distribution of charged particles for $70-80 \%$ most central bin in Au+Au collisions at $\sqrt{s_{N N}}=200 \mathrm{GeV}$. Net-charge (middle panel) and net-proton (right panel) distributions for $\mathrm{Au}+\mathrm{Au}$ collisions at $\sqrt{s_{N N}}=200 \mathrm{GeV}$ for three different centralities. The charged particle measurements are for $|\eta|<0.5$ and $0.15<p_{T}<1.0 \mathrm{GeV} / \mathrm{c}$ wheres the protons are within $|y|<0.5$ and $0.4<p_{T}<0.8 \mathrm{GeV} / \mathrm{c}$.

The left panel of Fig. 11 shows a typical $d E / d x$ vs. rigidity (defined as the momentum $\times$ the charge of the particle) distribution for charged particles as measured in the STAR TPC for 70$80 \%$ most central bin in $\mathrm{Au}+\mathrm{Au}$ collisions at $\sqrt{S_{N N}}=200 \mathrm{GeV}$. The figure gives a clean proton identification for $p_{T}<1 \mathrm{GeV} / c$. The net-charge $\left(h^{+}-h^{-}\right)$and net-proton $(p-\bar{p})$ distributions for $\mathrm{Au}+\mathrm{Au}$ collisions at $\sqrt{s_{N N}}=200 \mathrm{GeV}$ for three different centralities $(0-5 \%, 20-30 \%$ and 70-80\%) are also shown in Fig. 1. For the charged particles the distributions are symmetric around the mean value whereas the distributions for net-protons seem to be skewed in peripheral collisions. These distributions are further analyzed in order to extract various moments.

The characterization of a given data set may be obtained by analyzing the moments of their distribution. For a given distribution of $N$ data points, the third and fourth moments are expressed in terms of skewness and kurtosis as given by:

$$
\text { Skewness }=\frac{\left\langle(\delta N)^{3}\right\rangle}{\sigma^{3}} \text { and } \quad \text { Kurtosis }=\frac{\left\langle(\delta N)^{4}\right\rangle}{\sigma^{4}}-3 \text {, }
$$

where $\delta N=N-\langle N\rangle$ and $\sigma=\sqrt{\left\langle(\delta N)^{2}\right\rangle}$ is the standard deviation. Skewness is a measure of the symmetry of a distribution whereas kurtosis measures the peakedness of the distribution. For a Gaussian distribution, the values of skewness and kurtosis are zero. A positive value of skewness means a long tail on the right side and a negative value means the left tail is dominant. A data set with positive value of kurtosis is peaked at the center whereas a negative kurtosis is obtained for a flatter top near the mean. 

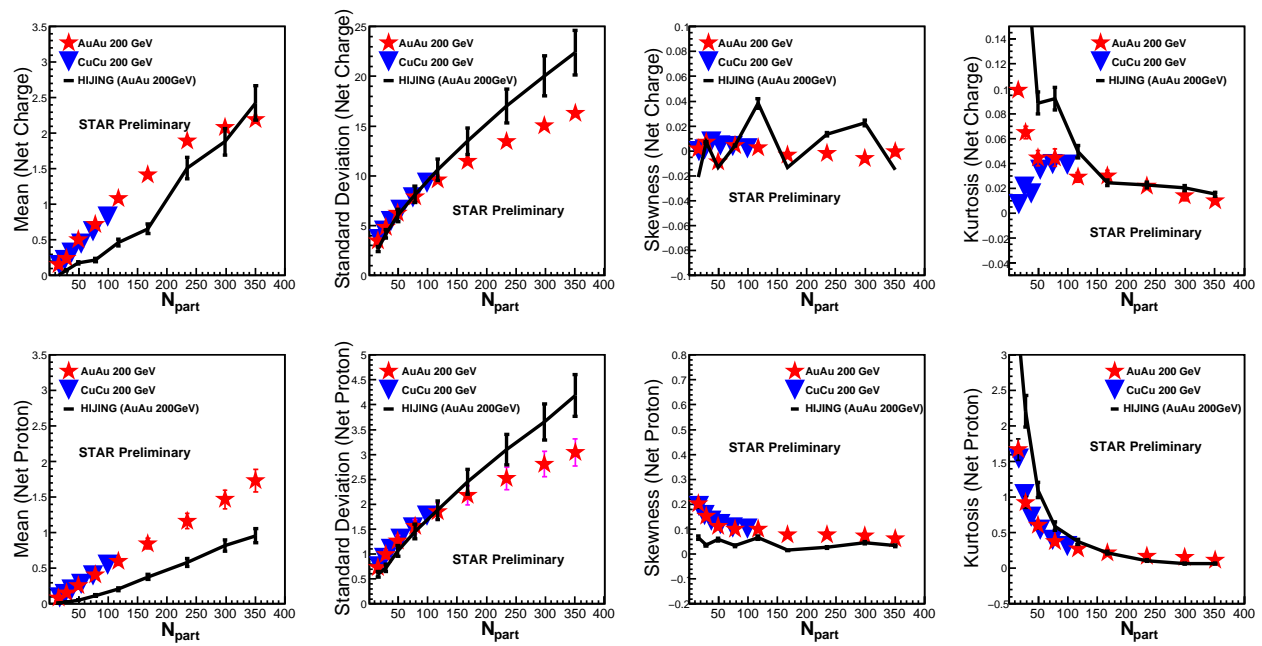

Figure 2: Mean, standard deviation, skewness and kurtosis of net-charge (top panels) and net-proton (bottom panels) distributions as a function of centrality for $\sqrt{s_{N N}}=200 \mathrm{GeV} \mathrm{Au}+\mathrm{Au}$ and $\mathrm{Cu}+\mathrm{Cu}$ collisions. HIJING results for $\mathrm{Au}+\mathrm{Au}$ $200 \mathrm{GeV}$ collision are also shown.

Fig. 2 shows the evolution of various moments of the net-charge and net-proton as a function of collision centralities (denoted by the number of participant nucleons, $N_{\text {part }}$ ) for $\mathrm{Au}+\mathrm{Au}$ and $\mathrm{Cu}+\mathrm{Cu}$ collisions at $\sqrt{s_{N N}}=200 \mathrm{GeV}$. Note that the distributions have not been corrected for reconstruction efficiency and acceptance. The error bars include both statistical and systematic errors obtained by varying track quality conditions. In going from peripheral to central collisions we observe that the mean and standard deviation increase smoothly for all cases. The skewness of net-charge distributions are close to zero and similar for all centralities, whereas for net-protons, the skewness values have a decreasing trend in going from peripheral to central collisions. For net-charge distributions, the kurtosis values are small and has a decreasing trend in going from peripheral to central collisions for $\mathrm{Au}+\mathrm{Au}$ systems. For net-protons, the higher values of kurtosis for peripheral collisions compared to central collisions indicate the distributions are more peaked at the mean value for peripheral collisions [13]. The results from HIJING event generator for $\mathrm{Au}+\mathrm{Au}$ at $200 \mathrm{GeV}$ are shown by solid lines, which are qualitatively similar to those of the data.

Several theoretical calculations based on Lattice QCD [9, 10, 11] and other QCD models [14] have suggested that the ratio of quartic to quadratic fluctuations of the net quark number, defined as $R_{4,2}$ to be a probe of QCD critical point. It can be shown that:

$$
R_{4,2}=\frac{\left\langle(\delta N)^{4}\right\rangle}{\left\langle(\delta N)^{2}\right\rangle}-3\left\langle(\delta N)^{2}\right\rangle=\text { Kurtosis } \times \text { Variance. }
$$

$R_{4,2}$ eliminates the $1 / \mathrm{N}$ behaviour necessary for the central limit theorem and removes explicit dependence on centrality. The significance of $R_{4.2}$ is that it is entirely dynamic and can be sensitive to the location of the critical point $[9,10,11,14]$.

Fig. 3 shows the $R_{4,2}$ values for net-charge and net-proton distributions. While the net proton values are close to unity, net-charge values are higher. The results are similar to those obtained from HIJING. While the HIJING event generator is not modelled on the existence of QCD critical point, its ability to reproduce the qualitative features of the data needs further investigation. 

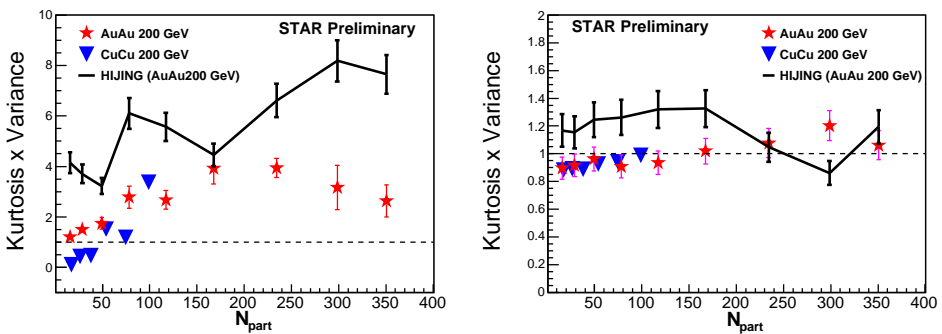

Figure 3: $R_{4,2}$ (= kurtosis $\times$ variance) of net-charge (left panel) and net-proton (right panel) distributions for $\sqrt{s_{N N}}=$ $200 \mathrm{GeV} \mathrm{Au}+\mathrm{Au}$ and $\mathrm{Cu}+\mathrm{Cu}$ collisions. Results from HIJING are shown as solid lines.

In summary, we have presented a study of mean, standard deviation, skewness and kurtosis of event-by-event net-charge and net-proton distributions in $\sqrt{s_{N N}}=200 \mathrm{GeV} \mathrm{Au}+\mathrm{Au}$ and $\mathrm{Cu}+\mathrm{Cu}$ collisions from the STAR experiment at RHIC. The results are presented as a function of collision centrality for mid-rapidity and low $p_{T}$ ranges. In case of net-charge distributions, the skewness values are close to zero for all centralities indicating the distributions are rather symmetric around the mean and the $R_{4,2}$ distributions are consistent with the predictions from HIJING. For netprotons, the kurtosis distributions are observed to decrease with increasing collision centrality and the $R_{4,2}$ is unity for all centrality bins. The values are in agreement with model calculations from HIJING which do not have the physics of QCD critical point. Detailed comparison with other event generators, such as UrQMD, is in progress [15].

The present study at the currently available RHIC energies only probes the $\mu_{B}<30 \mathrm{MeV}$ region of the phase diagram, while most theoretical calculations expect the QCD critical point to exist around $\mu_{B}>160 \mathrm{MeV}$. The current study will help to understand the expectations from various physics processes to the forthcoming RHIC critical point search program [4].

\section{Acknowledgments}

We thank Dr. S. Gupta, F. Karsch, V. Koch, K. Rajagopal, K. Redlich and M. Stephanov for exciting discussions on the subject.

\section{References}

[1] K. Rajagopal and F. Wilczek, The Condensed matter physics of QCD, arXiv:hep-ph/0011333

[2] C. Bernard et al., Phys. Rev. D 75, 094505 (2007).

[3] O. Scavenius, A. Mocsy, I. N. Mishustin and D. H. Rischke, Phys. Rev. C 64, 045202 (2001).

[4] B. I. Abelev et al., STAR Collaboration, STAR Internal Note - SN0493.

[5] R. Gavai and S. Gupta Phys. Rev. D 78, 114503 (2008).

[6] Z. Fodor and S.D. Katz JHEP 0404, 50 (2004).

[7] M. A. Stephanov et al., PRD 60, 114028 (1999).

[8] V. Koch, arXiv:0810.2520 [nucl-th].

[9] R. Gavai and S. Gupta, Phys. Rev. D 71, 114014 (2005).

[10] S. Ejiri et al., Nucl. Phys. Proc. Suppl. 140505 (2005).

[11] M. Cheng et al., arXiv:0811.1006 [hep-lat].

[12] M. A. Stephanov, Phys. Rev. Lett. 102 (2009) 032301.

[13] B. Mohanty et al., STAR Collaboration, Poster presented at Quark Matter 2009.

[14] Stokic, Friman and Redlich, Phys. Lett. B673, 192 (2009).

[15] T. Schuster et al., arXiv:0903.2911 [hep-ph]. 